\title{
James De Yoreo selected for 2016 David Turnbull Lectureship Award
}

$\mathrm{T}^{\mathrm{h}}$ he Materials Research Society's (MRS) David Turnbull Lectureship Award recognizes the career of a scientist who has made outstanding contributions to understanding materials phenomena and properties through research, writing, and lecturing, as exemplified by the late David Turnbull of Harvard University. This year, James De Yoreo, Chief Scientist for Materials Science at Pacific Northwest National Laboratory and Affiliate Professor of Materials Science and Engineering at the University of Washington, has been selected to give the 2016 Turnbull Lectureship. De Yoreo is cited "for transformational discoveries that have reshaped our understanding of crystallization science, achieved through the pioneering use of in situ microscopies and communicated to the world through eloquent lectures, insightful publications, and dedication to teaching professionals, students, and the general public." He will be presented with the award at the 2016 MRS Fall Meeting in Boston.

De Yoreo has reshaped decades-old theories and revealed previously unknown processes operating at the molecular level by pioneering the use of quantitative in situ microscopies to investigate the mechanisms of nucleation, crystal growth, and self-assembly. In his early work, he investigated the growth of single crystals from solution, defining a rigorous approach that provided solid quantitative data on step dynamics. He established the connection between atomic-scale events on crystal surfaces and macroscopic optical properties. He provided an explanation for the anomalous uniformity of growth rates despite the stochastic nature of growth sources, leading to the first technology for rapid growth of meter-scale optical crystals, for which he was awarded the Laudise Prize of the International Organization for Crystal Growth and received an R\&D 100 Award.

De Yoreo also revealed the capacity of simple ionic impurities to drive emergent phenomena of step dynamics, leading to ultrafast growth regimes, and showed how the multisite binding properties of biomolecules could give rise to bi-stability with spontaneous switching between growth modes and induced shape changes, including chirality in non-chiral crystals.

His work established the link between the stereochemical recognition of organic modifiers for atomic steps and changes in step-edge free energy and, ultimately, macroscopic shape. Extending this work to large peptides and full proteins, he established the role of peptide hydrophobicity in accelerating crystallization, and then used that principle to develop synthetic sequencedefined polymers that acted catalytically to achieve order-of-magnitude increases in rates. Having seen the importance of protein-crystal interactions in guiding crystal formation, De Yoreo directed his methods to the challenge of understanding how the proteins themselves assemble into the many ordered structures that are critical for the functions of living organisms, such as generation of mineralized tissues, creation of ion-selective membranes, and control over light and coloration.

He was the first to provide molecularscale proof for the two-step process of protein assembly. Watching assembly of bacterial membrane proteins, he directly imaged the formation of liquidlike clusters of unfolded proteins that fluctuated in structure until the ordered state emerged. Observing similar behavior during collagen assembly, he showed that the emergence of order is accompanied by accelerated rates of assembly. Through these studies, he proved that protein assembly mimics protein folding, with initial molten globular states, partially ordered intermediates, and well-ordered final states.

For his work on biomineralization and biomolecular assembly, De Yoreo received the highest award of the American Association for Crystal Growth and Epitaxy, as well as the LLNL Science and Technology Award. This work also formed the technical basis for his selection as a Fellow of the American Physical Society and a Fellow of MRS.

Over the past five years, De Yoreo has turned his attention to developing an understanding of nucleation phenomena in inorganic and biomineral systems, as well as post-nucleation growth by nanocrystal assembly. Pioneering the use of liquidphase transmission electron microscopy (TEM) for this purpose, he showed that a single system that exhibits multiple polymorphs can follow many pathways simultaneously for purely thermodynamic reasons. He also showed that a single pathway to the most stable phase can be selected by introducing low-energy interfaces. Using liquid-phase TEM, he then showed that by introducing highly charged polymers, which served as surrogates for macromolecules known to play a key role in biomineral formation, the pathway could again be redirected, instead forming the least stable phase for purely kinetic reasons.

As a result of his scientific achievements and contributions to the materials community, De Yoreo has been recognized with numerous awards, was recently elected to the Washington State Academy of Sciences, serves as chair of the MRS Program Development Subcommittee, and has provided classroom lectures on crystal growth in materials science, earth science, and physics around the country; he has trained more than 50 graduate students and postdocs in the US Department of Energy National Laboratory complex, and has authored, co-authored, or edited more than 220 publications and patents. 